\title{
DESIGN METHOD FOR A RECONFIGURABLE MECHANISM FOR FINGER REHABILITATION
}

\author{
Davin Sands \\ University of Idaho \\ dsands@vandals.uidaho.edu
}

\author{
Alba Perez Gracia \\ Idaho State University \\ Institut de Robotica i \\ Informatica Industrial (IRI), \\ UPC/CSIC \\ perealba@isu.edu
}

\author{
Jay McCormack \\ University of Idaho \\ mccormack@uidaho.edu
}

\author{
Eric T. Wolbrecht \\ University of Idaho \\ ewolbrec@uidaho.edu
}

\begin{abstract}
This paper presents a design method for a reconfigurable single degree-of-freedom mechanism for robotic assisted finger therapy following a stroke. The mechanism is a four-bar linkage that in combination with variable link lengths is capable of reproducing a power grasp finger motion for a wide variety of finger sizes. This is accomplished through an optimization procedure that determines the parameters of the four-bar linkage needed to fit the sampled range of finger trajectories. The linkage is located behind the hand and attaches to the medial phalanx of the finger just above the distal interphalangeal joint. In addition, the mechanism is designed so that it does not interfere with finger motion and so that the subject's fingertips and palm are free to touch real objects and experience tactile feedback. In future implementations, the mechanism could be used for a single finger or in parallel with other similar mechanisms to exercise multiple fingers simultaneously. Although the specific application presented here is the four-bar mechanism and finger power grasp motion, the developed design methods may be applied to a much broader range of mechanisms and applications where scalability for human-machine interface is required.
\end{abstract}

\section{KEY WORDS}

Rehabilitation robotics, design optimization, mechanism design

\section{Introduction}

Approximately 795,000 people suffer a stroke each year and $15 \%$ to $30 \%$ are disabled as a result [1]. Many are no longer able to participate in Activities of Daily Living (ADL) without assistance [2]. Most ADL's require common hand motions, such as pinching or grasping [3], and emotional well-being is directly, negatively influenced without the ability to perform these tasks [4]. Rehabilitation therapy is able to restore some of this functionality, but frequently people who have suffered a stroke do not have sufficient access to a therapist in order to make a significant recovery in these areas [1].
Repeated therapy with robots has shown promise in meeting the need for intensive, repetitive therapy [5] without constant, direct supervision by a therapist. Many devices currently exist which were either designed or can be used for rehabilitation of the hand [6]-[17]. The majority of this research focus has been on the robot design, with less attention given to evaluating control strategies for their ability to provide functional recovery. One open research question is how to best use robots in rehabilitation therapy to maximize functional recovery.

In order to answer that question, a control approach was designed in [18]-[20] based on the idea of increasing the effectiveness of robotic rehabilitation therapy by promoting neural-muscular participation of the subject. The developed "assist-as-needed" controller reduces assistance as the subject is able to complete a desired movement. In this way the subject cannot become dependent on the robot's assistance while still receiving enough assistance to complete the desired motion [18]. The controller was tested on Pneu-WREX, a robotic device designed for upper-limb rehabilitation [19], to evaluate its effectiveness in promoting neural-muscular recovery. The problem with this previous approach is that the complexity of the robot and relative low bandwidth of its control make it difficult to directly correlate controller performance to neural-muscular recovery. For this reason a more specialized, focused study of "assist-as-needed" control is desired. One focus area for such a study is the rehabilitation of the hand, which is critical to ADL's and has the potential to provide a more focused test of the relationship between control strategy and neuro-muscular recovery. We are therefore developing a device to test the "assist-as-needed" controller on the rehabilitation of the hand, specifically with respect to common finger motions, for either home or clinic use.

Previous robotic hand rehabilitation devices can be classified into two general categories: glove designs and exoskeleton designs. Of the glove designs, few allow for full tactile feedback for the palm, and many do not allow for tactile feedback for the fingertips either. Most also do not have drive systems that are backdrivable or have fast response [6], [7]. Of the exoskeleton designs, many have multiple degrees-of-freedom, and are therefore very 
dynamically complicated [8]-[13]. Some do not allow for the full range of finger motion [14]-[16], while others do not allow the motion to be sufficiently directed [15], [16]. Some use a cable drive system that induces significant friction, or have drive systems that are very stiff, and are therefore not adequately backdrivable [10]-[13]. Many also use attachment devices that do not leave the fingertips and/or palm open for tactile feedback [9], [10], [12]-[14], [16]. Some have features that can scale to different finger sizes [12], [13], [17], but they require many complex adjustments to affect a small change. The HWARD robot lacks many of the previously mentioned deficiencies [17], but is not simple to adjust, and it could not be easily redesigned to assist with motions other than the power grasp.

In order to provide an adequate test-bed for evaluating the effectiveness of various robotic rehabilitation therapies, the robotic device will need to meet certain key design criteria. Ideally, the robotic device should: 1) be highly backdrivable, so that the compliance is as completely determined by the controller as possible; 2) have a fast response and be capable of high accelerations, so that the control bandwidth is as high as possible; 3 ) allow tactile feedback of real objects for both the fingertips and palm, since such feedback would allow the subject to interact with a more natural environment, which may enhance recovery [17]; 4) be able to follow a specific predetermined trajectory, so that it can guide subjects through the desired motions; 5) be easily scalable to fit varying hand and finger sizes, so that a single design can be fabricated in bulk; and 6) be a compact size with a minimal number of actuators and attachments so that it is easy to use both at the clinic and/or at home. A device that meets these requirements can be dispensed to patients in their homes at minimal cost, allowing for large studies of the effectiveness of the device and controller to be conducted.

This paper presents a mechanism design approach for the development of a small, table-top hand rehabilitation device for use in the home and/or clinic. The design approach is able to produce a planar, single degree-offreedom mechanism design that is capable of tracking the finger during specific grasping motions. The resulting mechanism design will be used to design a device that will be driven by a linear voice coil actuator that is highly backdriveable and capable of high bandwidth operation. The design approach is also reconfigurable, including the ability to scale the mechanism design to a wide range of hand sizes, and can easily adapt the mechanism design to other hand motions.

Although the design approach presented here has been applied to a specific mechanism (four-bar) and a specific task (finger motion), its potential is much broader. This method is particularly advantageous for reducing the complexity of a robot (in terms of designing for fewer degrees-of-freedom) and finding the parameters that allow the device to be scalable for different trajectories of a specified motion.

\section{Methods}

A four step process was used to create a suitable mechanism design for a finger rehabilitation device. First, data was obtained that represented the trajectory that each finger travels through when completing the desired motion. Second, a planar mechanism type was chosen based on the trajectory complexity and the design equations were developed based on the kinematics of the selected mechanism. Third, optimization techniques were used to determine appropriate link lengths and angles for the mechanism that would follow the trajectories that were obtained. Fourth, based on the results of the previous optimization, the design was re-optimized to include the adjustment of link lengths and/or base point location of the mechanism that would enable it to track finger trajectories from a large number of different hand sizes. This four step process is described in the following sections.

\subsection{Collect Trajectories}

The mechanism being designed is intended to assist the hand through the power grasp motion. In order to collect trajectory data for that motion, a coordinate system and origin was defined with respect to the hand. Figure 1 below demonstrates this coordinate system along with the attachment point to the finger. The origin was placed at the center of the metacarpophalangeal joints so as to eliminate any motion of the wrist or hand. The $x$-axis was aligned with the back of the hand and arm, keeping the wrist straight, with the positive direction going out away from the metacarpophalangeal joints. The $y$-axis was set normal to the back surface of the hand, with the positive direction going out away from the back of the hand. The same coordinate system, as defined with respect to the hand, was used for both left and right handed individuals, thereby allowing data obtained from both hands could be compared directly for the optimizations.

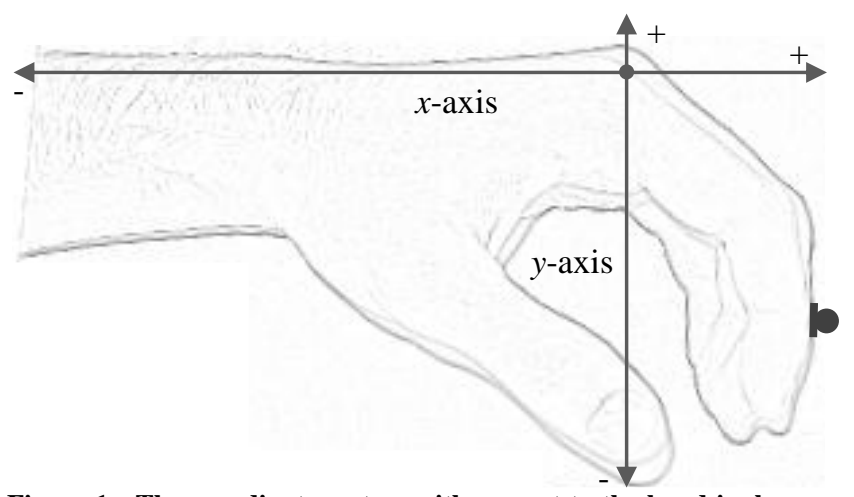

Figure 1: The coordinate system with respect to the hand is shown above. The attachment point to the finger on the medial phalanx just above the distal interphalangeal joint is also shown. The origin is marked with a dot.

Since maintaining tactile feedback for the finger is important in aiding recovery, the attachment point on the finger was fixed to the medial phalanx so that it would stay just above the distal interphalangeal joint (also shown in Figure 1. The trajectory data was collected by tracking 
the attachment point against the origin as defined in the coordinate system. The collected trajectory data only includes the planar motion of the finger, since the data was limited to the $x-y$ plane as defined in the coordinate system.

\subsection{Kinematics}

For this application, the desired mechanism was required to translate the motion of a one degree-offreedom actuator into the trajectories described by the collected data, which mandated a mechanism with only one degree-of-freedom. Since the collected trajectories could be accurately approximated by a fourth order polynomial (given that the orientation of the attachment to the finger was not specified), a four-bar mechanism was chosen for the design of the mechanism for this application. The variables that were defined for the fourbar mechanism are shown in Figure 2, where $\theta_{1-4}$ are the position angles and the remaining parameters (i.e., $O_{x}, O_{y}$, $\left.E_{x}, E_{y}, a, b, c, d, h, g, \alpha, \beta\right)$ are the structural variables of the mechanism.

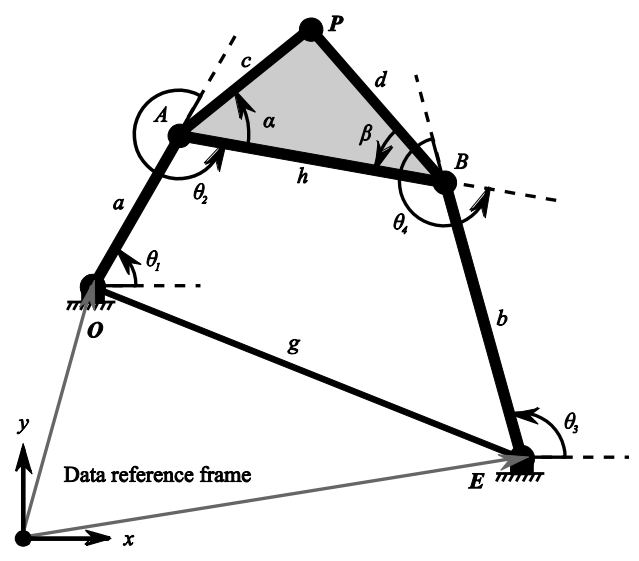

Figure 2: A generic four-bar linkage is shown above with the variables used in the optimizations labeled. $O, E$, and $P$ represent vectors from the origin to their respective points (the vector arrow for $P$ is omitted for clarity).

Using the variables defined in Figure 2, the forward kinematic equations for a planar four-bar mechanism are defined as [21], [22]:

$$
\begin{aligned}
& O_{x}+a \cdot \cos \left(\theta_{1}\right)+c \cdot \cos \left(\theta_{1}+\theta_{2}+\alpha\right)=P_{x} \\
& O_{y}+a \cdot \sin \left(\theta_{1}\right)+c \cdot \sin \left(\theta_{1}+\theta_{2}+\alpha\right)=P_{y} \\
& E_{x}+b \cdot \cos \left(\theta_{3}\right)-d \cdot \cos \left(\theta_{1}+\theta_{2}-\beta\right)=P_{x} \\
& E_{y}+b \cdot \sin \left(\theta_{3}\right)-d \cdot \sin \left(\theta_{1}+\theta_{2}-\beta\right)=P_{y}
\end{aligned}
$$

where point $P$ is the end-effector of the mechanism. Using the same definitions, the constraint equations for the planar four-bar mechanism are [21], [22],

$$
\begin{gathered}
g=\sqrt{\left(O_{x}-E_{x}\right)^{2}+\left(O_{y}-E_{y}\right)^{2}} \\
h=c \cdot \cos (\alpha)+d \cdot \cos (\beta) \\
K_{1}=E_{x}-O_{x}-a \cdot \cos \left(\theta_{1}\right) \\
K_{2}=E_{y}-O_{y}-a \cdot \sin \left(\theta_{1}\right) \\
K_{3}=\frac{b^{2}+K_{1}^{2}+K_{2}^{2}-h^{2}}{2 \cdot b} \\
\theta_{3}=\operatorname{atan} 2\left(K_{2}, K_{1}\right) \pm \operatorname{acos}\left(\frac{-K_{3}}{\sqrt{K_{1}^{2}+K_{2}^{2}}}\right) \\
\theta_{2}=\operatorname{atan} 2\left(\left[b \cdot \sin \left(\theta_{3}\right)+K_{2}\right],\left[b \cdot \cos \left(\theta_{3}\right)+K_{1}\right]\right)-\theta_{1}
\end{gathered}
$$

The constraint equations given above in (2) were used in conjunction with the forward kinematic equations in (1) and the structural variables of the mechanism to determine the trajectory that point $P$ would follow through the operation of the mechanism. This calculated trajectory was then compared to the collected finger trajectories to assess the tracking accuracy of the specific four-bar mechanism design.

\subsection{Single-Trajectory Optimization}

The next two steps in the design approach involve an optimization procedure that is shown in Figure 3 below. It illustrates the process that is described throughout the remainder of the methods section.

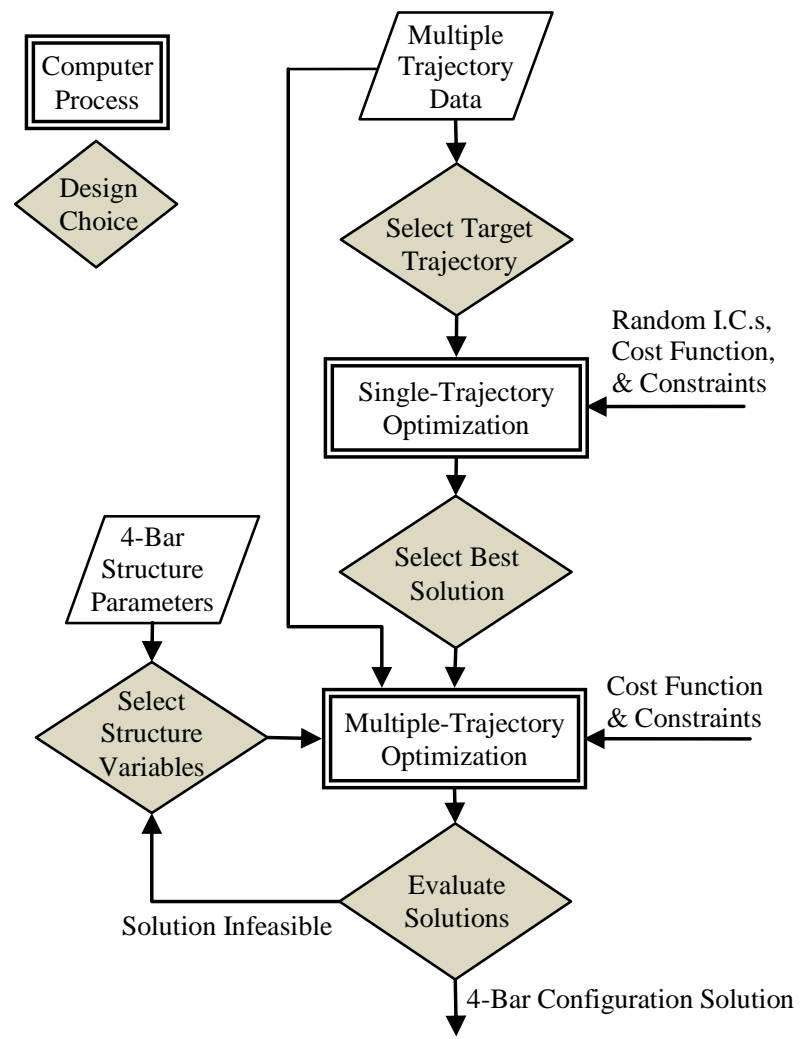

Figure 3: The flow chart above illustrates the optimization procedure, starting with the single-trajectory optimization which is used as the initial conditions for the final multiple-trajectory solution that can adjust to fit a range of finger trajectories. 
After defining the kinematic and constraint equations for the four-bar mechanism, the next step in the design was to optimize the kinematic equations with respect to a representative finger trajectory. Specifically, the mechanism parameters are optimized to follow a desired trajectory. This approach is discussed in [23]-[25] and others. For our application, a multivariable constrained minimization technique (MatLab ${ }^{\mathrm{TM}}$ function 'fmincon') was used to solve for the structural variables and position angles from the kinematic equations. This technique finds a local minimum for a single cost function based on a provided set of initial conditions, while forcing each variable to stay within a prescribed set of bounds or constraints. The cost function was derived by summing the square of the error between each data point in the finger trajectory and the specified position of the corresponding point on the mechanism based on the kinematic equations (represented by point $P$ ). This was accomplished by generating a set of kinematic equations with unique position angles for each data point in a selected finger trajectory. The $x$ and $y$ coordinate for each data point in the finger trajectory is represented by $p_{x}$ and $p_{y}$, respectively.

In addition to the squared error terms, two parameter constraints were added to the cost function to force the serial chain of each side of the four-bar mechanism to be unique, by imposing a preference towards non-zero positive values on the structural variables $h$ and $g$. The variables $\bar{h}$ and $\bar{g}$ in those parameter constraints represent the guesses made by the minimization function for the structural variables $h$ and $g$, respectively. By constraining $\bar{h}$ and $\bar{g}$ to a positive lower and upper bound, a penalty is introduced if the values for $h$ and $g$ exceed those bounds. The resulting cost function, $J$, with the kinematic expressions for $h$ and $g$ substituted in, is shown below in (3) where $n$ ranges from 1 to the number of data points in the trajectory.

$$
\begin{aligned}
J & =\sum_{n}\left\{\left[O_{x}+a \cdot \cos \left(\theta_{1, n}\right)+c \cdot \cos \left(\theta_{1, n}+\theta_{2, n}+\alpha\right)-p_{x, n}\right]^{2}\right. \\
& +\left[O_{y}+a \cdot \sin \left(\theta_{1, n}\right)+c \cdot \sin \left(\theta_{1, n}+\theta_{2, n}+\alpha\right)-p_{y, n}\right]^{2} \\
& +\left[E_{x}+b \cdot \cos \left(\theta_{3, n}\right)-d \cdot \cos \left(\theta_{1, n}+\theta_{2, n}-\beta\right)-p_{x, n}\right]^{2} \\
& \left.+\left[E_{y}+b \cdot \sin \left(\theta_{3, n}\right)-d \cdot \sin \left(\theta_{1, n}+\theta_{2, n}-\beta\right)-p_{y, n}\right]^{2}\right\} \\
& +[c \cdot \cos (\alpha)+d \cdot \cos (\beta)-\bar{h}]^{2} \\
& +\left[\sqrt{\left(O_{x}-E_{x}\right)^{2}+\left(O_{y}-E_{y}\right)^{2}}-\bar{g}\right]^{2}
\end{aligned}
$$

Upper and lower bounds were used to constrain each variable in the cost function. These bounds were:

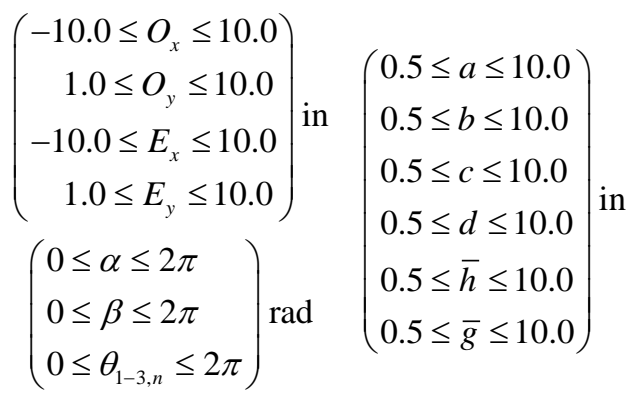

Two sets of constraints were used for the fixed base points (i.e., points $\boldsymbol{O}$ and $\boldsymbol{E}$, represented by variables $O_{x}$, $O_{y}, E_{x}$, and $E_{y}$ ), so that they could not interfere with the hand or finger motion. In the first set, they were constrained to be above the $x$-axis (positive $y$-coordinate) so that they would lie somewhere off the back of the hand. This constraint is demonstrated by the shaded area, A, in Figure 4. In the second set, they were constrained to be in front of the $y$-axis (positive $x$-coordinate) so that they would lie somewhere ahead of the fingers. This constraint is demonstrated by the shaded area, B, in Figure 4. The second set of constraints did not yield solutions that fit the design criteria, so the first set of constraints, as shown in (4), was used.

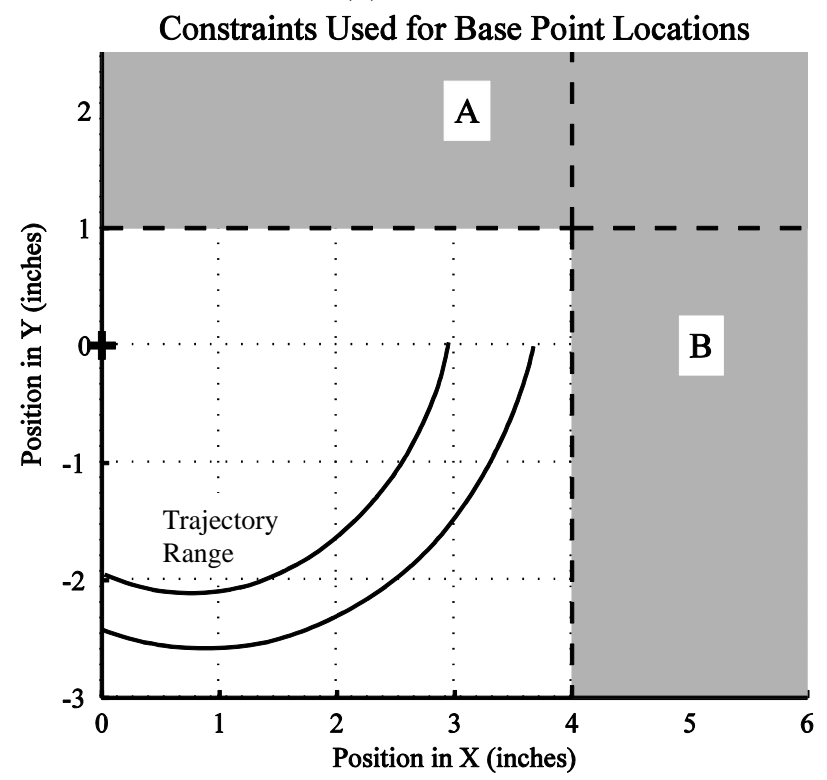

Figure 4: The base points were constrained to lie within the shaded regions shown above. The first set of constraints restricted the base points to region $A$, which extends farther to the left, right, and top. The second set of constraints restricted the base points to region $B$, which extends farther to the top, bottom, and right. The trajectory range is also shown. The origin is marked with a cross.

To arrive at an optimal solution within the bounds specified (a bounded global minimum rather than a local minimum), the initial conditions for each variable in the cost function were randomized between upper and lower bounds with an even distribution and solved iteratively with new random initial conditions for each iteration. All the solutions with a final cost function evaluation of less than a specified value were saved and evaluated 
qualitatively against a set of desired characteristics to select the optimal solution. In this case, the optimal solution was the one with a low cost function evaluation that had the shortest link lengths with the closest base point locations. This ensured that the resulting mechanism would require a minimal amount of material so that it would remain light weight, inexpensive, and easy to maneuver.

\subsection{Multiple-Trajectory Optimization}

Once an optimal solution was chosen based on a single-trajectory, the optimization cost function was expanded to include multiple trajectories from different hands and fingers in order to determine how to make the mechanism easily reconfigurable so that it would be able to fit trajectories from a large number of different hand sizes. This was accomplished by defining a select group of the structural variables that describe the mechanism as variable parameters in the cost function. These parameters were held constant for each individual trajectory, but were allowed to change between trajectories. All other structural variables in the mechanism were held constant for all trajectories, as in the previous optimization. The angles were unique for each data point in all trajectories, also as in the previous optimization. This modified the cost function by including a new variable for each parameter for each trajectory, which is shown in (5) below, with link lengths represented by variables $a$ and $b$ chosen as adjustable parameters, where $i$ ranges from 1 to the number of trajectories, and $n$ ranges from 1 to the number of data points in each trajectory.

$$
\begin{aligned}
J= & \sum_{i, n}\left\{\left[O_{x}+a_{i} \cdot \cos \left(\theta_{1, n, i}\right)+c \cdot \cos \left(\theta_{1, n, i}+\theta_{2, n, i}+\alpha\right)-p_{x, n, i}\right]^{2}\right. \\
& +\left[O_{y}+a_{i} \cdot \sin \left(\theta_{1, n, i}\right)+c \cdot \sin \left(\theta_{1, n, i}+\theta_{2, n, i}+\alpha\right)-p_{y, n, i}\right]^{2} \\
& +\left[E_{x}+b_{i} \cdot \cos \left(\theta_{3, n, i}\right)-d \cdot \cos \left(\theta_{1, n, i}+\theta_{2, n, i}-\beta\right)-p_{x, n, i}\right]^{2} \\
& \left.+\left[E_{y}+b_{i} \cdot \sin \left(\theta_{3, n, i}\right)-d \cdot \sin \left(\theta_{1, n, i}+\theta_{2, n, i}-\beta\right)-p_{y, n, i}\right]^{2}\right\} \\
& +[c \cdot \cos (\alpha)+d \cdot \cos (\beta)-\bar{h}]^{2} \\
& +\left[\sqrt{\left(O_{x}-E_{x}\right)^{2}+\left(O_{y}-E_{y}\right)^{2}}-\bar{g}\right]^{2}
\end{aligned}
$$

The variables that were allowed to change between trajectories ( $a$ and $b$ in this case) were selected by trial and error, where the desired outcome was the minimal group of variables that would still provide accurate trajectory tracking. This ensured that adjustments of the final mechanism to fit various hand sizes would be as simple as possible without compromising the ability to adjust to any size of hand within the range of sample trajectories used in the optimization. Variables that were the simplest to reconfigure from the viewpoint of manufacturability (e.g., a base point moving along a specified axis) were given preference over variables that were not as simple to reconfigure from one hand size to another (e.g., end-effector triangle dimensions).
With the variable parameters selected and multiple trajectories included, the cost function optimization was repeated with the same bounds used previously. The outcome from the single-trajectory optimization was used as initial conditions for this multiple-trajectory optimization, which yielded a multiple-trajectory solution (i.e., a scalable solution that includes multiple trajectories). In this way the multiple-trajectory solution was based on the selected single-trajectory solution. If a new form of solution was desired, it could be found more rapidly by performing random iterations of the singletrajectory optimization with modified bounds or new initial conditions to define the desired new form, and using that optimal solution as a basis for the multipletrajectory optimization, which would yield a new multiple-trajectory solution in the desired new form.

\section{Results}

For the preliminary design, eight power grasp finger trajectories were collected. These trajectories came from the index and middle fingers of four different hands, two of which were female, and two of which were male. Three of these finger trajectories which represent the range of all eight finger trajectories are shown in Figure 5. The corresponding calculated trajectories generated by the linkage are also shown for comparison.

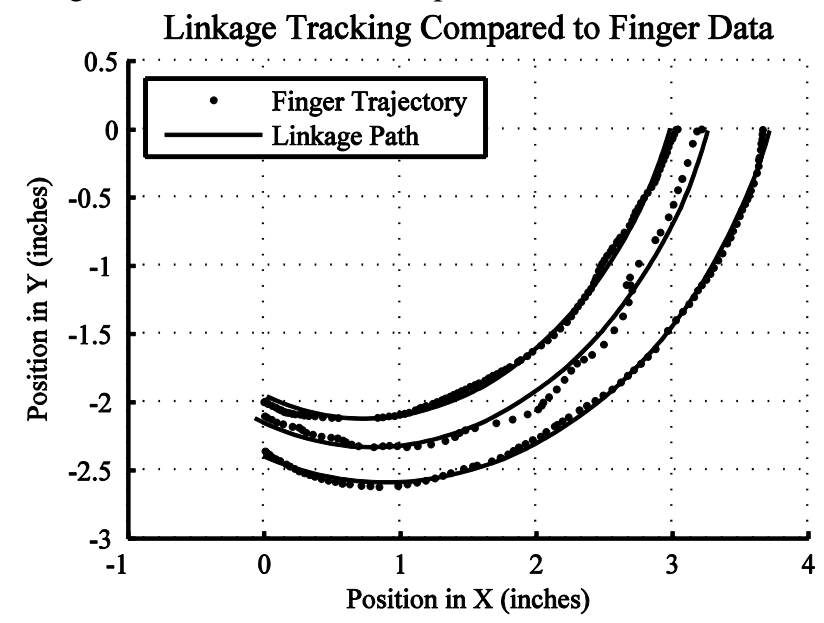

Figure 5: The three trajectories shown above represent the range of the eight trajectories used in this design optimization. These trajectories show the path of a point just off the distal interphalangeal joint as the finger moves through a power grasp motion. The calculated trajectory generated by the linkage for each corresponding finger trajectory is also shown.

\subsection{Single-Trajectory Solution}

A representative trajectory from the sample described above was used to optimize all the structural variables in the four-bar linkage to generate and select a singletrajectory solution through the procedures outlined in the methods above. The bounds used to constrain each variable are listed in (4) in section 2 above. Out of the generated solution possibilities, the solution described below was selected due to the short link lengths and close base point locations. The values for the structural variables of the four-bar linkage that were obtained in the 
selected single-trajectory solution are displayed below in (6). The variable names refer to those on the diagram of the four bar linkage shown previously in Figure 2.

$$
\left(\begin{array}{l}
O_{x}=0.601 \\
O_{y}=1.982 \\
E_{x}=0.968 \\
E_{y}=1.199
\end{array}\right) \text { in }\left(\begin{array}{l}
a=4.409 \\
b=4.207 \\
c=2.797 \\
d=2.377
\end{array}\right) \text { in }\left(\begin{array}{l}
\alpha=5.330 \\
\beta=4.424
\end{array}\right) \mathrm{rad}
$$

The resulting design and a few different positions along the motion of the resulting four-bar linkage are depicted in Figure 6 below. The root-sum-square error of the trajectory of this linkage compared to the original finger trajectory was 0.079 inches.

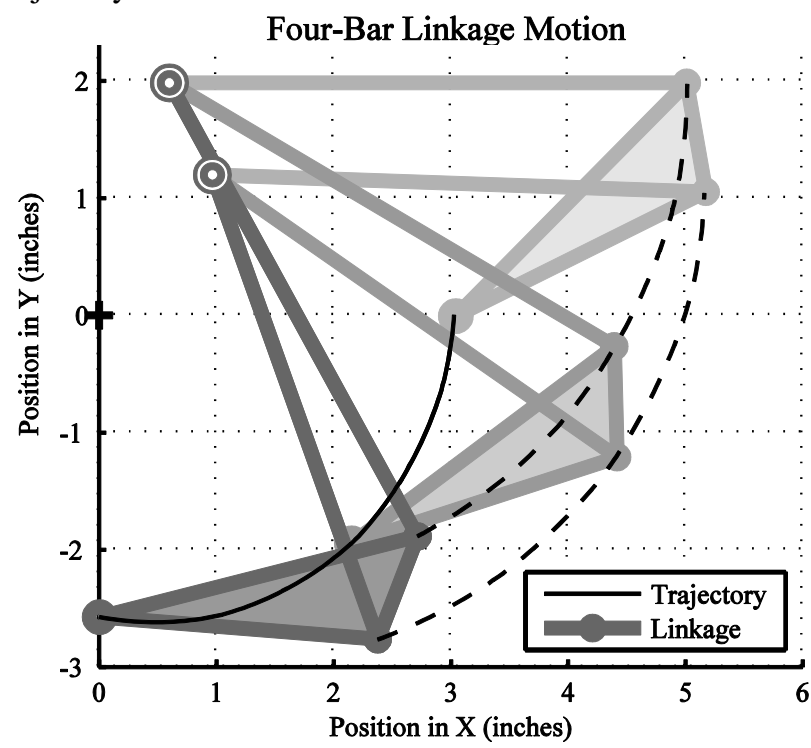

Figure 6: The four-bar linkage described by the selected singletrajectory solution is shown above in three different positions throughout the motion as the resulting trajectory is generated. The dashed lines represent the path taken by the other two joints of the linkage. The origin is marked with a cross.

\subsection{Multiple-Trajectory Solution}

After obtaining an optimized solution for the single finger trajectory as shown above, the remaining finger trajectories were included in a new cost function along with the original finger trajectory to obtain a new solution that would be scalable to the entire group of collected finger trajectories through the procedures previously discussed. The values from the initial optimization given in (6) were used as initial conditions for this second optimization. The bounds used were the same as in the previous optimization. The link lengths $a$ and $b$ were selected to be the reconfigurable variable parameters between each trajectory. These variables were chosen because they allowed enough variability in the four-bar linkage to cover all the collected finger trajectories while keeping the linkage small and relatively close to the hand and without significantly increasing the complexity of the linkage from a manufacturability standpoint. Other combinations of variables were tried, as specified in the methods above, but they did not result in a linkage with these desirable characteristics.
The values for the structural variables that were obtained for all of the four-bar linkages in the selected multiple-trajectory solution are:

$$
\left(\begin{array}{l}
O_{x}=1.154 \\
O_{y}=2.690 \\
E_{x}=1.429 \\
E_{y}=1.312
\end{array}\right) \text { in }\left(\begin{array}{l}
c=2.249 \\
d=1.545
\end{array}\right) \text { in }\left(\begin{array}{l}
\alpha=5.592 \\
\beta=4.330
\end{array}\right) \mathrm{rad}
$$

The values that were obtained for link lengths $a$ and $b$ for each four-bar linkage in the optimized multiple-trajectory solution are displayed in (8) below. The subscripted numbers on the variables $a$ and $b$ indicate the trajectory to which they are associated.

$$
\begin{aligned}
a_{1-8}= & \{4.268,4.258,4.238,4.004, \\
& 3.633,3.351,3.264,3.217\} \text { in } \\
b_{1-8}= & \{3.841,3.849,3.817,3.562, \\
& 3.302,3.034,3.011,2.934\} \text { in }
\end{aligned}
$$

The range of designs for the corresponding four-bar linkages for the range of finger trajectories is depicted in Figure 7 below.

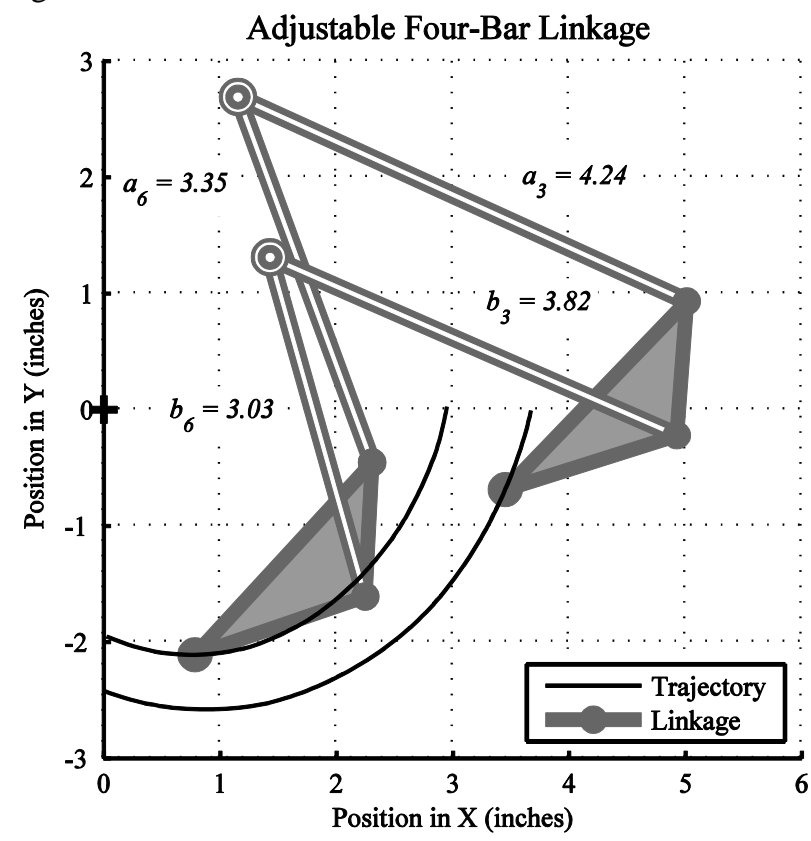

Figure 7: Two configurations and corresponding trajectories of four-bar linkages determined by the multiple-trajectory solution are shown above. The only difference between linkages is the length of the links represented by variables $a$ and $b$. Other configurations are omitted for clarity. The origin is marked with a cross.

The root-sum-square error for the tracking of the calculated trajectory of each linkage compared to the corresponding finger trajectory is shown in (9) below. The RSS error for the original single-trajectory solution was 0.079 inches.

$$
\begin{aligned}
\text { RSS Error }_{1-8}=\{ & 0.245,0.193,0.249,0.487, \\
& 0.411,0.398,0.260,0.217\} \text { in }
\end{aligned}
$$

The tracking errors for each trajectory shown in (9) are relatively small, indicating that the four-bar linkage 
described by this multiple-trajectory solution is capable of correctly directing each sampled finger through the prescribed motion by simply manipulating the link lengths $a$ and $b$. This solution is also capable by the same means of adjusting to any other hand size that is in the range of the trajectories that were sampled above.

\section{Conclusions and Discussion}

This paper describes a method for designing a fourbar linkage that can produce a specific finger motion trajectory and is reconfigurable to a variety of hand sizes. The resulting reconfigurable mechanism design will be part of the design of a rehabilitation robot that will be used to evaluate the "assist-as-needed" controller [18] for rehabilitation of the hand. This novel design method could also be applied to other single or multiple degreesof-freedom mechanisms, and may be used to design a mechanism that interfaces with the hand and fingers that is connected to a larger mechanism for combined rehabilitation of the upper limbs and hands, allowing for robotic rehabilitation of a wide range of upper body motions required for activities of daily living.

The solution presented here shows the efficacy of the presented method and specifically demonstrates how it is applied. Trajectories that represent the full range of the desired motion were collected, and one trajectory from that range was used iteratively in a single-trajectory optimization with random initial conditions until a singletrajectory solution is generated that meets the design criteria. This solution was then used as initial conditions along with all the collected trajectories and a chosen set of variable parameters in the multiple-trajectory optimization. The final multiple-trajectory solution includes the ability to reconfigure the mechanism to any trajectory within the provided range by mechanically changing the length of two links.

The solution produced by these methods satisfies each of the design criteria. The mechanism design is highly backdrivable and allows for high control bandwidth with the selection of a suitable actuator. Tactile feedback is allowed due to the choice of attachment point location on the finger. The mechanism design follows each of the sampled trajectories with low error, and is simply adjusted for each trajectory by manipulating only two parameters. The entire final device including the required single actuator would sit very close to the hand and arm, minimizing the final size and weight.

A final implementation of the developed method would require a larger sample size of finger trajectories in order to adequately represent the hand sizes that the mechanism would need to accommodate. A suitable design for hand rehabilitation can be obtained by following the procedures and methods presented here with a large enough sample of finger trajectories.

After a reconfigurable linkage design has been produced based on a suitable sample range, a specific finger measurement (e.g., medial phalanx length) from the subjects of the original sample may be used to correlate between that metric of finger size and the reconfigurable values for the link lengths. This would provide a prescribed way to interpolate the appropriate parameters for a given subject, and would be independent of whether or not that subject's fingers were a part of the original sample (so long as their hand size fit within the range of the original sample).

The methods outlined here could also be used to adapt an existing four-bar linkage to a new set of bounds or constraints (this would be independent of whether or not the existing four-bar linkage was created using these methods). This would be useful for the situation where a mechanism-based robotic hand rehabilitation device needed to be redesigned to eliminate interference with an attachment to an upper limb rehabilitation device, for example. This would also be useful for the situation where such a device designed for assistance with the power grasp motion needed to be redesigned for assistance with a different motion (such as the lateral pinch motion). In this last case, new sample trajectories of the new motion would also need to be collected.

Future work includes designing and creating a prototype of a robotic hand rehabilitation device based on the presented mechanism design methods. This includes selecting a four-bar linkage solution based on a full sample range and completing the kinematic analysis of that linkage. In addition, constraints representing power transmission should be added to the design analysis to further optimize the design for force transmittance. An actuator would then be selected based on the force profiles that would be powerful enough while still maintaining the high compliance needed to allow kinematic errors [20]. Other sensors would also be added as required by the controller. Finally, a robotic hand rehabilitation device would be designed based on the selected four-bar linkage solution that incorporates a practical adjustment scheme for the variable parameters that has enough resolution to cover the subjects' variability while still maintaining fabrication and adjustment simplicity and rigidity.

The method developed here was created for the express purpose of designing a mechanism for a robotic hand rehabilitation device. However, it encompasses a path generation design tool for any generic reconfigurable multi-bar linkage, and can therefore be used in the design and optimization of a linkage for any application where the motion of a multi-bar mechanism is suitable and where a reconfigurable multi-bar linkage design is needed to cover a large number of similar trajectories with minimal adjustment. This is particularly useful in applications that include a human-machine interface.

\section{References}

[1] D. Lloyd-Jones, R. J. Adams, T. M. Brown, M. Carnethon, S. Dai, G. De Simone, T. B. Ferguson, E. Ford, K. Furie, C. Gillespie, A. Go, K. Greenlund, N. Haase, S. Hailpern, P. M. Ho, V. Howard, B. Kissela, S. Kittner, D. Lackland, L. 
Lisabeth, A. Marelli, M. M. McDermott, J. Meigs, D. Mozaffarian, M. Mussolino, G. Nichol, V. L. Roger, W. Rosamond, R. Sacco, P. Sorlie, R. Stafford, T. Thom, S. Wasserthiel-Smoller, N. D. Wong, J. Wylie-Rosett, Heart Disease and Stroke Statistics-2010 Update: A Report From the American Heart Association, Circulation 121, 2010, e46-e215.

[2] S. Stephens, R. A. Kenny, E. Rowan, R. N. Kalaria,M. Bradbury, R. Pearce, K. Wesnes, C. G. Ballard, Association Between Mild Vascular Cognitive Impairment and Impaired Activities of Daily Living in Older Stroke Survivors Without Dementia, Journal of the American Geriatrics Society, 53(1), 2005, 103-107.

[3] O. Bozo, C. A. Guarnaccia, Activities of Daily Living, Social Support, and Future Health of Older Americans, The Journal of Psychology, 144(1), 2010, 1-14.

[4] J. A. Katt, L. Speranza, W. Shore, K. H. Saenz, E. L. Witta, Doing Well: A SEM Analysis of the Relationships Between Various Activities of Daily Living and Geriatric Well-Being, The Journal of Genetic Psychology, 170(3), 2009, 213-226.

[5] E. M. Frick, J. L. Alberts, Combined Use of Repetitive Task Practice and an Assistive Robotic Device in a Patient With Subacute Stroke, Physical Therapy, 86(10), 2006, 1378-1386.

[6] D. E. Nathan, M. J. Johnson, J. R. McGuire, Design and validation of low-cost assistive glove for hand assessment and therapy during activity of daily living-focused robotic stroke therapy, Journal of Rehabilitation Research \& Development, 46(5), 2009, 587-602.

[7] H. C. Fischer, K. Stubblefield, T. Kline, X. Luo, R. V. Kenyon, D. G. Kamper, Hand Rehabilitation Following Stroke: A Pilot Study of Assisted Finger Extension Training in a Virtual Environment, Topics in Stroke Rehabilitation, 14(1), 2007, 1-12.

[8] S. Itoa, H. Kawasakia, Y. Ishigureb, M. Natsumec, T. Mouria, Y. Nishimotod, A design of fine motion assist equipment for disabled hand in robotic rehabilitation system, J. Franklin Inst. (2009), doi:10.1016/j.jfranklin.2009.02.009.

[9] T. Koyama, I. Yamano, K. Takemura, T. Maeno, Multi-Fingered Exoskeleton Haptic Device using Passive Force Feedback for Dexterous Teleoperation, Proc. IEEE/RSJ Intl. Conf. On Intelligent Robots and Systems, Lausanne, Switzerland, 2002, 2905-2910.

[10] A. Wege, K. Kondak, G. Hommel, Mechanical Design and Motion Control of a Hand Exoskeleton for Rehabilitation, Proc. IEEE ICMA Intl. Conf. on Mechatronics and Automation, 2005, 155-159.

[11] M. J. Lelieveld, T. Maeno, T. Tomiyama, Design and Development of Two Concepts for a 4 DOF Portable Haptic Interface with Active and Passive Multi-Point Force Feedback for the Index Finger, Proc. of IDETC/CIE Intl Design Engineering
Technical Conferences \& Computers and Information in Engineering Conference, Philadelphia, PA, 2006, 1-10.

[12] J. Wang, J. Li, Y. Zhang, S. Wang, Design of an Exoskeleton for Index Finger Rehabilitation, Proc. $31^{\text {st }}$ IEEE EMBS Intl. Conf. of the Engineering in Medicine and Biology Society, Minneapolis, MN, 2009, 5957-5960.

[13] T.T. Worsnopp, M.A. Peshkin, J.E. Colgate, D.G. Kamper, An Actuated Finger Exoskeleton for Hand Rehabilitation Following Stroke, Proc. $10^{\text {th }}$ IEEE Intl. Conf. on Rehabilitation Robotics, Noordwijk, The Netherlands, 2007, 896-901.

[14] E. J. Koeneman, R. S. Schultz, S. L. Wolf, D. E. Herring, J. B. Koeneman, A Pneumatic Muscle Hand Therapy Device, Proc. $26^{\text {th }}$ IEEE EMBS Intl. Conf. of the Engineering in Medicine and Biology Society, San Francisco, CA, 2004, 2711-2713.

[15] L. Dovat, O. Lambercy, R. Gassert, T. Maeder, T. Milner, T. C. Leong, E. Burdet, HandCARE: A Cable-Actuated Rehabilitation System to Train Hand Function After Stroke, IEEE Transactions on Neural Systems and Rehabilitation Engineering, 16(6), 2008, 582-591.

[16] M. Bouzit, G. Burdea, G. Popescu, R. Boian, The Rutgers Master II - New Design Force-Feedback Glove, IEEE/ASME Transactions on Mechatronics, 7(2), 2002, 256-263.

[17] C. D. Takahashi, L. Der-Yeghiaian, V. H. Le, S. C. Cramer, A Robotic Device for Hand Motor Therapy After Stroke, Proc. $9^{\text {th }}$ IEEE Conf. on Rehabilitation Robotics, Chicago, IL, 2005, 17-20.

[18] E. T. Wolbrecht, V. Chan, D. J. Reinkensmeyer, J. E. Bobrow, Optimizing Compliant, Model-Based Robotic Assistance to Promote Neurorehabilitation, IEEE Transactions on Neural Systems and Rehabilitation, 16(3), 2008, 286-297.

[19] E. T. Wolbrecht, V. Chan, V. Le, S. C. Cramer, D. J. Reinkensmeyer, J. E. Bobrow, Real-time computer modeling of weakness following stroke optimizes robotic assistance for movement therapy, Proc. $3^{\text {rd }}$ IEEE EMBS Intl. Conf. on Neural Engineering, Kohala Coast, HI, 2007, 152-158.

[20] D. J. Reinkensmeyer, J. A. Galvez, L. Marchal, E. T. Wolbrecht, J. E. Bobrow, Some Key Problems for Robot-Assisted Movement Therapy Research: A Perspective from the University of California at Irvine, Proc. $10^{\text {th }}$ IEEE Intl. Conf. on Rehabilitation Robotics, Noordwijk, The Netherlands, 2007, 10091015.

[21] R. M. Murray, Z. Li, S. S. Sastry, A Mathematical Introduction to Robotic Manipulation (CRC Press, 1994).

[22] J. M. McCarthy, Geometric Design of Linkages (Springer, 2000). 